\title{
1 Borrelia infection in rodent host has dramatic effects on the microbiome of ticks
}

3 Phineas T. Hamilton ${ }^{1 *}$, Elodie Maluenda ${ }^{2}$, Anouk Sarr $^{2}$, Alessandro Belli $^{3}$, Georgia Hurry $^{4}$,

4 Olivier Duron ${ }^{5,6}$, Olivier Plantard ${ }^{7}$, Maarten J. Voordouw ${ }^{2,4^{*}}$

$6 \quad{ }^{1}$ Deeley Research Centre, BC Cancer, Victoria BC, Canada

$7 \quad{ }^{2}$ Laboratory of Ecology and Evolution of Parasites, Institute of Biology, University of

8 Neuchâtel, Neuchâtel, Switzerland.

$9{ }^{3}$ Laboratory of Ecology and Epidemiology of Parasites, Institute of Biology, University of

10 Neuchâtel, Neuchâtel, Switzerland.

$11{ }^{4}$ Department of Veterinary Microbiology, Western College of Veterinary Medicine, University

12 of Saskatchewan, Saskatoon, Canada.

$13{ }^{5}$ Centre of Research in Ecology and Evolution of Diseases (CREES), Montpellier, France

$14{ }^{6}$ MIVEGEC (Maladies Infectieuses et Vecteurs: Ecologie, Génétique, Evolution et Contrôle),

15 Univ. Montpellier (UM) - Centre National de la Recherche Scientifique (CNRS) - Institut pour la

16 Recherche et le Développement (IRD), Montpellier, France

$17{ }^{7}$ INRAE, Oniris, BIOEPAR, 44300, Nantes, France

$19{ }^{*}$ Corresponding author email: phin.hamilton@gmail.com; maarten.voordouw@usask.ca

21 Keywords: Borrelia, dysbiosis, Ixodes, Lyme disease, microbiome, tick-borne disease, vector 


\section{Abstract:}

24 Background: Vector-borne diseases remain major causes of human morbidity and mortality. It

25 is increasingly recognized that the community of microbes inhabiting arthropods can strongly

26 affect their vector competence, but the role of the tick microbiome in Borrelia transmission - the

27 cause of Lyme disease - remains unclear.

29 Results: Here, we use a large-scale experiment to clarify the reciprocal interactions between

30 Borrelia afzelii and the microbiome of Ixodes ricinus, its primary vector. In contrast to other

31 reports, we find that depletion of the bacterial microbiome in larval ticks has no effect on their

32 subsequent acquisition of $B$. afzelii during blood feeding on infected mice. Rather, exposure to $B$.

33 afzelii-infected hosts drives pervasive changes to the tick microbiome, decreasing overall

34 bacterial abundance, shifting bacterial community composition, and increasing bacterial

35 diversity. These effects appear to be independent of the acquisition of B. afzelii by ticks,

36 suggesting they are mediated by physiological or immunological aspects of $B$. afzelii infection in

37 the rodent host.

39 Conclusions: Manipulation of the microbiome of I. ricinus larvae had no effect on their ability

40 to acquire B. afzelii. In contrast, B. afzelii infection in the mouse had dramatic effects on the

41 composition of the gut microbiome in I. ricinus nymphs. Our study demonstrates that vector-

42 borne infections in the vertebrate host shape the microbiome of the arthropod vector. 


\section{Background}

Infectious diseases vectored by arthropods impose an enormous burden on human health [1-3]. For successful transmission, however, vector-borne pathogens must contend with both the

47 immunological defenses of the arthropod vector, and the community of other microbiota that

48 inhabit it [4-7], and it is now clear that endogenous microbes can shape the competence of

49 diverse arthropod vectors in acquiring and transmitting pathogens [8-12]. Experimental

50 perturbations of the microbiome ('dysbiosis') via the use of antibiotics or other methods have, in

51 various contexts, been shown to either increase or decrease the susceptibility of arthropods to

52 colonization by vector-borne pathogens $[8,10,12,13]$. In particular, the introduction of the

53 intracellular bacterium Wolbachia into mosquito vectors [14] can dramatically reduce mosquito

54 competence to vector arboviruses, malaria parasites, and filarial nematodes (Kambris et al. 2009,

55 Moreira et al. 2009, Bian et al. 2010). These effects appear to be mediated through the innate

56 immune system of the arthropod host, rendering the mosquito less susceptible to infection by

57 diverse pathogens [15-17]. Similarly, Enterobacter bacteria in the midgut of Anopheline

58 mosquitoes produce reactive oxygen species that can kill malaria parasites [9]. Such direct and

59 indirect antagonistic interactions have formed the basis of increasingly sophisticated biological

60 control strategies that include the ongoing use of Wolbachia to control Dengue virus

61 transmission by Aedes mosquitoes [3, 14, 18, 19].

Hard ticks remain among the most important vectors of infectious disease in the northern

63 hemisphere, transmitting numerous pathogens that include the causative agents of Lyme

64 borreliosis ('Lyme disease'), anaplasmosis, babesiosis, and tick-borne encephalitis [20-23]. In

65 particular, the increasing incidence of Lyme and other tick-borne diseases in parts of Europe and

66 North America has underscored the public health risks associated with hard ticks [24-28]. Recent 
67 work on Ixodes scapularis has suggested that perturbations to the tick's microbiome can

68 influence tick susceptibility to B. burgdorferi sensu stricto (ss) and Anaplasma phagocytophilum

69 by affecting the integrity of the tick midgut $[8,12]$, although the generality and importance of

70 these effects in the natural transmission of these pathogens are unclear. It is likewise unclear if

71 and when specific members of the tick microbiota can interfere with Borrelia colonization of the

72 tick, or if Borrelia impacts the tick microbiota in ways that might affect the dynamics of other

73 tick-borne diseases.

74 Here, we use a large-scale experiment with tick offspring derived from wild-collected

75 gravid tick mothers to investigate reciprocal interactions between Borrelia afzelii, an endemic

76 cause of Lyme disease in Europe [29], and the microbiome of Ixodes ricinus, its primary vector.

77 We found that disrupting the microbiome of larval I. ricinus ticks by bleaching egg casings has

78 profound yet transient effects on the tick microbiota, but no effect on subsequent colonization by

79 B. afzelii when ticks feed on B. afzelii-infected hosts. In contrast, feeding on B. afzelii-infected

80 mice has pervasive effects on the composition and diversity of the tick microbiome, which are

81 largely independent of prior microbiome disruption. This suggests that B. afzelii infection

82 dynamics within rodent hosts have strong potential to sculpt the tick microbiota, with unclear

83 consequences for the transmission of other tick-borne pathogens. Negative interactions between

84 B. afzelii and other members of the tick microbiome might provide insights into the biological

85 control of tick-borne diseases.

\section{$87 \quad$ Methods}

Borrelia, ticks, and mice: We used Borrelia afzellii isolate NE4049, which was obtained

89 from an I. ricinus nymph at a field site near Neuchâtel, Switzerland. This strain has multi-locus 
90 sequence type ST679, ospC major group allele A10, and strain ID number 1887 in the Borrelia

91 MLST database. We used isolate NE4049 because we have previously shown that it is highly

92 infectious for both mice and ticks [30-32]. Pathogen-free, female Mus musculus BALB/c ByJ

93 mice were used as the vertebrate reservoir host. To infect mice via tick bite, we used Ixodes

94 ricinus nymphs infected with isolate NE4049 that had been generated in a previous study [30]

95 and that came from our laboratory colony of Borrelia-free I. ricinus ticks. For these I. ricinus

96 nymphs, the percentage of nymphs infected with B. afzelii ranged between $80.0 \%$ and $100.0 \%$.

97 Uninfected control nymphs were obtained from our laboratory colony of Borrelia-free I. ricinus

98 ticks.

Experiment: The experimental design of the study is shown in Figure 1. Engorged adult

100 female I. ricinus ticks were collected from wild roe deer captured in the Sylve d'Argenson forest

101 near Chizé, France. The female ticks were allowed to lay their eggs in the laboratory. Four weeks

102 after deposition, each of the 10 clutches of eggs was split into two batches. One batch was rinsed

103 with $10 \%$ bleach while the other batch was rinsed with water (Figure 1). The rinsed eggs were

104 allowed to hatch into larvae under non-sterile conditions. To test whether the bleach treatment

105 had reduced the microbiota in the larval ticks, a group of $\sim 400$ larvae was frozen for each of the

10620 batches at six weeks after hatching.

107 The remaining larvae for each of the 20 batches were split into two groups of $\sim 100$

108 larvae. For each of the 20 batches of eggs (10 tick families x 2 egg washing treatments), one

109 group of larvae was fed on an uninfected control mouse ( $\mathrm{n}=20$ control mice) whereas the other

110 group of larvae was fed on a $B$. afzelii-infected mouse $(n=20$ infected mice; Figure 1). These

111 mice had been infected with B. afzelii (or not) via nymphal tick bite (see below for details). The

112 resultant engorged larvae were placed in individual Eppendorf tubes and allowed to moult into 
113 nymphs. Four weeks after the larva-to-nymph moult, 10 nymphs were randomly selected from

114 each mouse and were frozen at $-80^{\circ} \mathrm{C}$ (Figure 1). In summary, we froze 400 nymphs $(10$

115 families*2 egg washing treatments* 2 mice infection statuses*10 nymphs/mouse).

116 Experimental infection of mice: For the main experiment, $40 \mathrm{BALB} / \mathrm{c}$ mice were

117 randomly assigned to either the control group or the infection group. Each mouse in the control

118 group $(\mathrm{n}=20)$ was infested with 5 uninfected I. ricinus nymphs, whereas each mouse in the

119 infected group $(\mathrm{n}=20)$ was infested with 5 B. afzelii-infected I. ricinus nymphs. Each of the 40

120 mice had been infested with 5 nymphs, so that each mouse had similar immune experience with

121 ticks. Five weeks after the nymphal challenge, an ear tissue biopsy and a blood sample were

122 taken from each of the 40 mice. The ear tissue biopsy was tested for the presence of B. afzelii

123 infection using qPCR. The blood sample was tested for Borrelia-specific antibodies using a

124 commercial Lyme disease ELISA. These tests confirmed that the 20 mice in the infected group

125 were infected with B. afzelii, whereas the 20 mice in the control group were uninfected. These 40

126 mice were used to feed the larval ticks (see above and in Figure 1).

127 Molecular methods for larval ticks: The 20 groups of larval ticks that had been frozen

128 were split into two sub-groups with 200 larval ticks per sub-group. Half of these sub-groups

129 were washed with ethanol prior to DNA extraction and the other sub-groups were not washed.

130 DNA extraction of the 40 sub-groups of larval ticks was done using a QIAGEN kit following the

131 manufacturer's instructions. The DNA of each sub-group was eluted into $100 \mu$ of distilled

132 water. The DNA concentration was measured for each of the 40 sub-groups using a Nanodrop.

133 For qPCR, the DNA concentration of each sub-group was adjusted to $5 \mathrm{ng} / \mu \mathrm{l}$. Two qPCR assays

134 were performed independently for each DNA extraction: tick calreticulin and bacterial 16S

$135 r R N A$. Each qPCR assay contained $3 \mu \mathrm{l}$ of template for a total of $15 \mathrm{ng}$ of DNA. 
Molecular methods for nymphal ticks: For each mouse, the 10 nymphs were split into

137 two groups of 5 nymphs. Ticks in one group were washed with ethanol prior to DNA extraction

138 and ticks in the other group were not washed. DNA extraction of whole ticks was done using a

139 QIAGEN kit following the manufacturer's instructions. The DNA of each tick was eluted into 65

$140 \mu \mathrm{l}$ of distilled water. The DNA concentration was measured for each of the 400 nymphs using a

141 Nanodrop. For qPCR, the DNA concentration of each tick was adjusted to $5 \mathrm{ng} / \mu \mathrm{l}$. Three qPCR

142 assays were performed independently for each DNA extraction: tick calreticulin, bacterial $16 S$

$143 r R N A$, and Borrelia flagellin (see electronic supplementary material (ESM) for details). Each

144 qPCR assay contained $3 \mu \mathrm{l}$ of template for a total of $15 \mathrm{ng}$ of DNA.

146 nymphs for which we had quantified bacterial load using the $16 S$ rRNA gene qPCR assay,

147 Illumina sequencing was performed for 360 nymphs. Sample preparation consisted of two PCR

148 reactions. In the first reaction we amplified a $464 \mathrm{bp}$ fragment of the V3-V4 region of the $16 \mathrm{~s}$

149 rRNA gene using primers Bakt_341F (5'CCTACGGGNGGCWGCAG-3') and Bakt_805R

150 (5'GACTACNVGGGTATCTAATCC-3') [33], designed with Illumina adapters. Reactions were

151 performed in a final volume of $50 \mu \mathrm{l}$ using $2.5 \mathrm{U}$ of HotStar HiFidelity DNA polymerase

152 (Qiagen,Germany), $2.5 \mu 1$ of $10 \mu \mathrm{M}$ primers, $10 \mu 1$ of $15 \mu \mathrm{M}$ dNTP mix, with a thermal cycle

153 with a denaturation step of $95^{\circ} \mathrm{C}$ for $5 \mathrm{~min}, 45$ cycles of $94^{\circ} \mathrm{C}$ for $15 \mathrm{sec}, 51^{\circ} \mathrm{C}$ for 45 seconds,

154 and $72^{\circ} \mathrm{C}$ for 45 seconds, with a final elongation step at $72^{\circ} \mathrm{C}$ for 7 minutes. Amplicons were

155 purified with the Wizard SV Gel and PCR Clean-Up system (Promega Switzerland).

156 The second PCR incorporated the sample barcodes. Reactions were performed in a final

157 volume of $25 \mu \mathrm{l}$ using $1.25 \mathrm{U}$ of HotStar HiFidelity DNA polymerase, $1 \mu \mathrm{l}$ of $10 \mu \mathrm{M}$ primers, 5

$158 \mu \mathrm{l}$ of $15 \mu \mathrm{M}$ dNTP mix. The thermocycler had a denaturation step of $95^{\circ} \mathrm{C}$ for $5 \mathrm{~min}, 12$ cycles 
159 of $95^{\circ} \mathrm{C}$ for $30 \mathrm{sec}, 55^{\circ} \mathrm{C}$ for 30 seconds, and $72^{\circ} \mathrm{C}$ for 30 seconds, with a final elongation step of

$16072^{\circ} \mathrm{C}$ for 5 minutes, and amplicons purified as above. The 360 purified amplicons were pooled in

161 equimolar concentration using a Qubit 2.0 fluorometer (Invitrogen) and sequenced by

162 Microsynth (Balgach, Switzerland) using an Illumina MiSeq v2 with $250 \mathrm{bp}$ paired end output,

163 followed by adaptor and quality trimming.

165 Statistical Methods

Analysis of the bacterial load in the larvae: For each sub-group of larval ticks, we

167 divided the $16 S$ rRNA gene copy number by the calreticulin gene copy number. These $16 S$ rRNA

168 to calreticulin ratios were $\log 10$-transformed to improve the normality of the data. The $\log 10$ -

169 transformed $16 S$ rRNA to calreticulin ratios were analyzed using linear mixed effects models

170 (LMMs). Fixed factors included egg washing treatment (2 levels: water and bleach), larval tick

171 washing treatment (2 levels: none and ethanol), and their interactions. Random factors included

172 tick family. We used R/Bioconductor (v 3.4.2. or above) for analyses, including the lme4,

173 complexHeatmap, vegan, and phyloseq packages [34-37].

174 Data selection of the nymphs: Of the 370 DNA extractions, 14 were not included in the

175 analysis because their DNA concentrations were too low. After adjustment of the DNA

176 concentration, the remaining 356 DNA extractions had a DNA concentration that ranged

177 between 3.33 and $5.00 \mathrm{ng} / \mu \mathrm{l}$ so that the $3 \mu \mathrm{l}$ of DNA template contained between 10.0 and 15.0

178 ng of DNA.

179 Analysis of the bacterial load in the nymphs: For each tick, we divided the $16 S r R N A$

180 gene copy number by the calreticulin gene copy number. These ratios were $\log 10$-transformed to

181 normalize the data. The $\log 10$-transformed $16 S$ rRNA to calreticulin ratios were analysed using 
182 LMMs. Fixed factors included egg washing treatment (2 levels: water and bleach), B. afzelii

183 infection status of the mouse (2 levels: uninfected control, infected), nymphal tick washing

184 treatment (2 levels: none and ethanol), and their interactions. Random factors included tick

185 family and mouse identity nested inside tick family.

Analysis of the B. afzelii infection prevalence in the nymphs: These analyses were

187 restricted to the subset of nymphs that had fed as larvae on the B. afzelii-infected mice. We used

188 a proportion test to determine whether the microbiome reduction of the egg washing treatment

189 influenced the susceptibility of the nymphs to acquire $B$. afzelii infection during the larval blood

190 meal. The nymphal infection status $(0=$ uninfected, $1=$ infected $)$ was also analysed using

191 generalized linear mixed effects models (GLMMs) with binomial errors. Fixed factors included

192 egg washing treatment, nymphal tick washing treatment, and their interaction. Random factors

193 included tick family and mouse identity nested inside tick family.

Analysis of the B. afzelii spirochete load in the nymphs: This analysis was restricted to

195 the subset of nymphs that were infected with B. afzelii. For each tick, we divided the B. afzelii

196 flagellin gene copy number by the tick calreticulin gene copy number. These ratios were log10-

197 transformed to normalize the data. The log10-transformed flagellin to calreticulin ratios were

198 analysed using LMMs. Fixed factors included egg washing treatment, nymphal tick washing

199 treatment, and their interaction. Random factors included tick family and mouse identity nested

200 inside tick family.

Analysis of 16S rRNA amplicons: Because of limited overlap between the 250 bp read

202 ends, we elected to use an OTU picking strategy that did not require first assembling paired end

203 reads into contigs. We generated OTU tables using the CD-HIT-OTU-Miseq workflow [38]

204 packaged with CD-HIT v 4.6.8. Forward and reverse read lengths were specified at 200 and 150 
205 bp and clustered against the SILVA 132 99\% OTU release [39], otherwise using default

206 parameters that included using Trimmomatic for read trimming [40]. This identified 10,454

207 OTUs, although the vast majority of reads (93.6\%) recruited to the 100 most abundant OTUs. It

208 also assigned taxonomy to only 926 OTUs (<10\%): for more robust taxonomic assignments, we

209 applied Metaxa2 v2.2 [41] to the representative sequences for each OTU identified by CD-HIT

210 using default parameters and the included reference database in Metaxa2. This identified 7,550

211 OTUs as bacterial and provided taxonomies that were largely congruent with CD-HIT

212 assignments, where evaluable; subsequent analyses were restricted to this bacterial OTU set.

213 The OTU table and taxonomy were imported to R (>v. 3.4.2), and analyzed using the

214 phyloseq, vegan, and DeSeq2 packages (as detailed in Results). Linear mixed models for

215 univariable outcomes were implemented using the lme4 package with a family as a random

216 effect, or nesting mouse host within tick family. In analyses using $D e S e q 2$ and $d b-R D A$, family

217 was included as a fixed factor (DeSeq2) or a conditioning variable ( $d b-R D A)$. We evaluated the

218 reliability of our replicated sequencing/analysis approach on the same nymphal samples via the

219 variance explained by sample in $d b-R D A$, and the intraclass correlation coefficient for Shannon

220 diversity. For statistical analyses requiring tick infection status, we imputed missing infection

221 status for one Borrelia-exposed nymph as infected, as that was the most common state of

222 exposed nymphs.

\section{Results}

225 Egg bleaching profoundly disrupts the microbiome of tick larvae

226 Egg bleaching had strong effects on the abundance of bacteria associated with tick larvae, 227 as shown by a 27.5-fold reduction in the relative $16 S$ rRNA gene (hereafter $16 S$ ) copy number 
228 (scored via qPCR) in larvae hatching from bleached versus unbleached eggs $(\mathrm{N}=29$ evaluable

229 samples; linear mixed model (LMM) controlling for tick family; $\mathrm{P}<10^{-6}$; $\log _{10}$ ratio of

23016 S/calreticulin 0.24 versus 6.61, respectively; Figure 2A). This reduction was evident six

231 weeks after treatment, demonstrating a profound effect of bleaching on the larval tick

232 microbiome. In contrast, washing larvae with ethanol prior to DNA extraction, which is expected

233 to reduce the external microbiota [but see 42], did not have a significant effect on the $16 S$ copy

234 number (LMM; $\mathrm{P}=0.12$; Figure 2A). Taken together, these observations suggest that the egg

235 bleaching-induced reduction of $16 S$ copy number was driven by an increase in the relative

236 abundance of the internal microbiota of the larval ticks at the expense of the external microbiota.

237 Multivariate analysis of the larval tick microbiome using db-RDA revealed that egg

238 bleaching led to a clear shift in the $16 \mathrm{~S}$ community, whereas there was no discernible effect of

239 washing the larvae prior to DNA extraction (db-RDA; based on Bray-Curtis dissimilarities of

$240 \log _{10}$ OTU abundances, stratified by tick family. Permutation tests; $\mathrm{P}<0.001$ and 0.727 ,

241 respectively; Figure 2B). Consistent with our expectations, the most significantly

242 (proportionally) enriched taxon due to bleaching was in the order Rickettsiales, which we further

243 manually annotated to Candidatus Midichloria mitochondrii, an endosymbiont of I. ricinus that

244 we expect would be unaffected by external bleaching (Figure 2C; $\mathrm{P}_{\text {adj }}<10^{-6}$; shown are the $\log _{10}$

245 read counts per thousand mapped reads). In contrast, egg bleaching significantly reduced the

246 relative abundance of Pseudomonas OTU 23 in the resultant larvae, which suggests that this

247 bacterium is found on the surface of the eggshell (Figure $2 \mathrm{C} ; \mathrm{P}_{\mathrm{adj}}<10^{-3}$. Other significant

248 effects associated with bleaching included an increase in Methylobacterium, a taxon that has

249 been implicated as a potential contaminant of laboratory reagents [43]; these are consistent with

250 the strong reduction in $16 S$ abundance we observed via qPCR. No OTUs changed significantly 
251 from washing (all $\mathrm{P}_{\mathrm{adj}}>0.05$ ). In sum, egg bleaching dramatically decreased $16 S$ copy number

252 and shifted the microbial community composition in larvae measured at 6 weeks after treatment.

253 Egg bleaching probably reduced the relative abundance of bacteria associated with the egg

254 surface and thereby increased the relative abundance of endosymbiont bacteria. The lack of an

255 observed effect of washing the larvae — on both microbial abundance and microbial diversity-

256 suggests that external microbiota are a minor component of the $16 S$ diversity in the lab-hatched

257 tick larvae.

Manipulation of the larval microbiome has no effect on the acquisition of B. afzelii, and the microbiome largely recovers in the unfed nymphs

261 We screened nymphs for B. afzelii infection via qPCR and found no evidence that 262 disruption of the larval microbiome affected either the percentage of ticks that acquired the 263 infection while feeding (with infection prevalences of $68.2 \%$ and $73.5 \%$ in unbleached and

264 bleached groups respectively; binomial GLMM; $\mathrm{P}=0.44, \mathrm{~N}=183$, Figure 3A) or B. afzelii copy

265 number in the nymphs that became infected (LMM; $\mathrm{P}=0.958, \mathrm{~N}=130$; Figure 3B). Thus,

266 although the egg bleaching treatment was highly effective at reducing the microbiome in larvae,

267 it did not affect the ability of B. afzelii to colonize ticks during or following the larval blood 268 meal.

269 Although the egg bleaching treatment strongly reduced the $16 S$ copy number in the 270 larvae, the bacterial community largely recovered after these larvae had taken a blood meal and 271 molted into nymphs, as there was no effect of the egg bleaching on $16 S$ copy number in nymphs

272 (LMM; P = 0.272, $\mathrm{N}=356$, Figure 3C). Intriguingly, in contrast to the non-significant effect of 273 ethanol washing on $16 S$ copy number that we observed in larvae, there was a clear reduction 
274 with both washing and B. afzelii exposure. The $16 S$ copy number was lowest in ticks that were

275 both washed and B. afzelii-exposed (LRT for interaction; $\mathrm{P}<10^{-5}$ ), indicating that feeding on $B$.

276 afzelii-infected mice reduced the internal bacterial load in nymphs; we investigate this hypothesis

277 in more detail below.

Host infection with B. afzelii has pervasive effects on the microbiome of I. ricinus nymphs

To extend our analysis of larval ticks to the nymphal stage, we used $16 \mathrm{~S}$ amplicon

281 sequencing to profile replicate nymphs from each treatment alongside the larval samples (above).

282 We again restricted these analyses to the 40 most abundant OTUs. As expected from prior

283 reports $[44,45]$, there was a clear shift in the tick-associated bacterial community from larval to

284 nymphal ticks (Figure 4A). There was, further, a striking effect of B. afzelii infection in the

285 mouse on the tick microbiota as quantified in the nymphal stage, with pronounced differences

286 between ticks fed on B. afzelii-infected versus control mice (db-RDA; P < 0.001; Figure 4B

287 shows unsupervised principal coordinates analysis (PCoA)). We also found that egg bleaching

288 and ethanol washing prior to DNA extraction had modest but significant effects on microbiome

289 composition in nymphs $(\mathrm{P}<0.001$ and $\mathrm{P}=0.014$, respectively; Figure 4B), consistent with our

290 finding that washing the nymphs decreased $16 S$ copy number. While sequencing recovered

291 OTUs annotated as Borrelia, the most abundant Borrelia-annotated OTU was ranked $81^{\text {st }}$ in

292 overall abundance and accounted for $<0.05 \%$ of total sequence reads. Thus, a direct contribution

293 of sequenced Borrelia $16 S$ amplicons in infected ticks does not explain these strong patterns.

294 Microbiome $\alpha$-diversity (measured as Shannon entropy) in nymphs modestly increased as

295 a result of both microbiome disruption via egg bleaching (LMM; $\mathrm{P}=0.035 ; \mathrm{N}=305$. Figure

296 5A) and from B. afzelii infection $(\mathrm{P}=0.0025$; Figure 5B $)$ or exposure $(\mathrm{P}=0.0007$; competing 
297 multivariable model; Figure 5B). Coupled with the decrease in $16 S$ copy number observed when

298 feeding on B. afzellii-infected mice, these diversity effects appeared to be mediated through

299 disproportionately negative impacts on abundant OTUs (e.g., Stenotrophomonas) leading to

300 increased community evenness. As mentioned, these trends were evident when considering the

301 infection status of the tick itself, or that of the mouse on which they were fed (Figure 5C).

302 Comparison between control nymphs, uninfected nymphs, and infected nymphs demonstrated

303 that it was feeding on an infected mouse rather than acquiring B. afzelii infection that was most

304 important for determining the nymphal microbiome (Figure 5C). This was supported by

305 comparing competing models with Akaike's Information Criterion (AIC), which found that

306 feeding on an infected mouse was a much stronger predictor of both $\alpha$-diversity $(\triangle \mathrm{AIC} \sim 6)$ and

307 the multivariate bacterial community (Figure 5A; $\triangle \mathrm{AIC} \sim 14$ in models using first PCoA axis as

308 response variable) than acquisition of $B$. afzelii by the tick (Figure 5), suggesting that the effects

309 we observe are more likely to be caused by physiological or immunological characteristics of

310 infected mice rather than the direct effects of B. afzelii infection in the ticks. Similarly,

311 examining the OTU frequencies in bleached and B. afzelii-exposed ticks showed that these net

312 effects were driven by proportional reductions in the dominant OTUs, with concomitant

313 increases in less frequent OTUs (e.g. Figure 4). Collectively, these results are consistent with the

314 strong effects of mouse B. afzelii infection status we observe on $16 S$ copy number and suggest

315 that these are specifically mediated by disproportionate negative effects on abundant bacterial

316 OTUs. 
320 exposure, we used negative binomial models implemented in phyloseq/DESeq2. In line with the

321 global diversity shifts we observed, feeding on $B$. afzelii-infected mice led to significant changes

322 in the relative frequencies of many OTUs (with 19/40 significant at $\mathrm{P}_{\mathrm{adj}}<0.05$; Figure 5). This

323 was most evident in large decreases in the frequency of multiple Stenotrophomonas OTUs and

324 other Gammaproteobacteria (Figure 6). As a result, there appeared to be a degree of taxonomic

325 dependence in microbial responses to B. afzelii exposure, with Betaproteobacteria generally

326 significantly increasing in frequency and Gammaproteobacteria decreasing (Fisher's exact test, P

$327=0.004$; Figure 6). Our analysis also revealed that several nymph OTUs that increased in

328 frequency in response to tick bleaching, including two Burkholderia OTUs (Betaproteobacteria;

$329 \mathrm{P}_{\mathrm{adj}}<0.01$; Figure 6) and a Bradyrhizobium OTU (Alphaproteobacteria; $\mathrm{P}_{\mathrm{adj}}<0.01$; Figure 6),

330 whereas no OTUs significantly (proportionally) decreased. Consistent with our expectations,

331 ethanol washing prior to DNA extraction increased the relative abundance of the endosymbiotic

332 Candidatus Midichloria mitochondrii (annotated as order Rickettsiales, Alphaproteobacteria) as

333 well as Spiroplasma (Mollicutes) by 2.5 and 1.7-fold respectively $\left(\mathrm{P}_{\mathrm{adj}}<0.01\right.$ ), corroborating an

334 enriching effect of washing on the internal tick microbiota.

\section{Discussion}

Here, we used a highly replicated experiment on wild-collected tick families to examine

338 the reciprocal interactions between the endogenous tick microbiota and B.afzelii. We used egg

339 bleaching to radically disrupt the microbiome in larval ticks but found no evidence that this

340 affected the subsequent susceptibility of ticks to infection with B. afzelii. Rather, this work

341 revealed striking effects of feeding on B. afzelii-infected mice on the tick microbiome; these 
342 effects superseded those of actually acquiring an infection, as judged by comparing competing

343 statistical models, and the resultant partitioning of samples in microbial community space (e.g.,

344 Figure 4A, 5C).

345 A growing number of studies have investigated whether dysbiosing ticks influences their

346 susceptibility to acquiring tick-borne pathogens [8, 11-13]. In some systems, microbiome

347 disruption makes tick species more susceptible to infection with tick-borne pathogens [8, 13],

348 whereas other systems found the opposite effect $[11,12]$. In the present study, we found no

349 evidence that microbiome disruption influenced $B$. afzelii infection rates or the B. afzelii

350 spirochete load in infected ticks. In contrast, a previous study found that dysbiosed I. scapularis

351 larvae were less likely to acquire B. burgdorferi ss and contained lower bacterial loads compared

352 to control larvae [12]. These two studies differed in a number of factors including the Borrelia

353 species, the Ixodes tick species, and the method of dysbiosis. An important aspect of the present

354 study is that we investigated whether dysbiosis of the eggs influenced the infection status of the

355 nymphs, which is the stage that is actually critical for the transmission of Lyme disease in nature 356 [46].

357 Despite the fact that bleaching the eggs was highly effective at reducing the bacterial

358 microbiome in the resultant larvae, this method of 'dysbiosis' did not have a meaningful impact

359 on B. afzelii transmission. While the presence of additional bacteria to those uncovered here

360 could influence these patterns, we found little influence of tick family on the recovered bacterial

361 communities, outside of potentially vertically transmitted bacteria such as Spiroplasma,

362 suggesting the microbial community of I. ricinus is largely homogenous at the scale studied here.

363 Our method of dysbiosis further highlights the neglected importance of maternal transmission of

364 gut symbionts in ticks. In arthropods, gut symbionts are typically vertically transmitted by 
365 superficial bacterial contamination of eggs (egg smearing) [47]. Our egg washing with bleach

366 has removed such maternally inherited gut symbionts and this impacted the microbial

367 communities hosted by larvae. Our study shows that egg smearing is a key mechanism for

368 colonization of ticks by their associated microbes.

B. afzelii infection reduced the microbial abundance (in the ethanol-washed nymphs) and

370 changed the microbial community in the unfed nymphs. Notably, we observed changes in OTU

371 relative abundance to be, at least in part, taxon-specific with decreases in Gammaproteobacteria

372 and increases in Betaproteobacteria (Figure 6). The observation that mouse infection status was

373 more important than tick infection status suggests that the blood physiology at the time of the

374 larval blood meal was critical for structuring the subsequent nymph microbiome. Metabolomic

375 studies of mouse serum samples have shown that B. burgdorferi ss infection changes the blood

376 concentration of amino acids, energy metabolites, and aromatic compounds [48], which could

377 influence the development of the tick microbiome. Infection with B. burgdorferi sl stimulates the

378 host immune system, which could also exert collateral damage on the tick microbiome [49-52].

379 For example, elevated levels of complement, cytokines, leukocytes, and reactive oxygen species

380 in the blood [52-55] may interact inside the tick to have negative effects on the midgut

381 microbiome. In summary, our study suggests that the physiological and immunological changes

382 associated with infection in the vertebrate host have important consequences for the microbiome

383 of feeding ticks.

\section{Conclusions}

386 In summary, we found that egg bleaching resulted in a 30-fold reduction of the microbiome of

387 larval ticks. This microbiome manipulation had no effect on the ability of larval ticks to acquire 
B. afzelii after feeding on infected mice. Once the engorged larvae had moulted into unfed nymphs, the dramatic effect of the egg bleach treatment on the tick microbiome had mostly disappeared. The B. afzelii infection status of the mice that provided the larval blood meal had a

391 dramatic effect on the microbiome of the resultant unfed nymphs. Our study suggests that

392 infection in the vertebrate host influences the quality of the larval blood meal with long-term

393 consequences for the tick microbiome that persist into the nymphal stage.

Ethics approval and consent to participate: The commission that is part of the "Service de la

397 Consommation et des Affaires Vétérinaires (SCAV)" of Canton Vaud, Switzerland evaluated

398 and approved the ethics of this study. The Veterinary Service of the Canton of Neuchâtel,

399 Switzerland issued the animal experimentation permit used in this study (NE04/2014).

Availability of data and materials: Raw sequencing reads will be deposited at NCBI (accession

402 pending). Supplementary data and scripts to reproduce the analysis will be made available at

403 github.com/onecarbon/tickdysbiosis (pending).

404

405 Competing interests: The authors declare that they have no competing interests.

406

407 Funding: This work was supported by the following grants awarded to Maarten J. Voordouw: a

408 Swiss National Science Foundation grant (FN 31003A_141153) and a Discovery Grant from the

409 Natural Sciences and Engineering Research Council of Canada (RGPIN-2019-04483). PTH was

410 supported by a Canadian Institutes for Health Research PDF. 
412 Authors' contributions: EM, OD, OP and MJV conceived and designed the study. EM, AS, and

413 AB conducted the experiment and performed the molecular work. PTH and MJV analysed the

414 data and wrote the manuscript. GH created the figure of the experimental design. All authors

415 read and approved the final version of the manuscript.

417 Acknowledgments: We thank Gilles Capron (Office National de la Chasse et de la Faune

418 Sauvage) and the Office National des Forêts of the Réserve biologique domaniale intégrale de la

419 Sylve d'Argenson for permission to collect ticks on roe deer captured in the Chizé forest

420 (France). 
1. Jones KE, Patel NG, Levy MA, Storeygard A, Balk D, Gittleman JL, et al. Global trends in emerging infectious diseases. Nature (Lond). 2008;451:990-4.

2. Kilpatrick AM, Randolph SE. Drivers, dynamics, and control of emerging vector-borne zoonotic diseases. Lancet. 2012;380(9857):1946-55; doi: 10.1016/s0140-6736(12)611519.

3. McGraw EA, O'Neill SL. Beyond insecticides: new thinking on an ancient problem. Nat Rev Microbiol. 2013;11(3):181-93; doi: 10.1038/nrmicro2968.

4. Bonnet SI, Binetruy F, Hernandez-Jarguin AM, Duron O. The tick microbiome: why non-pathogenic microorganisms matter in tick biology and pathogen transmission. Front Cell Infect Microbiol. 2017;7; doi: 10.3389/fcimb.2017.00236.

5. de la Fuente J, Antunes S, Bonnet S, Cabezas-Cruz A, Domingos AG, Estrada-Pena A, et al. Tick-pathogen interactions and vector competence: identification of molecular drivers for tick-borne diseases. Front Cell Infect Microbiol. 2017;7; doi: 10.3389/fcimb.2017.00114.

6. Narasimhan S, Fikrig E. Tick microbiome: the force within. Trends Parasitol. 2015;31(7):315-23; doi: 10.1016/j.pt.2015.03.010.

7. Weiss B, Aksoy S. Microbiome influences on insect host vector competence. Trends Parasitol. 2011;27(11):514-22; doi: 10.1016/j.pt.2011.05.001.

8. Abraham NM, Liu L, Jutras BL, Yadav AK, Narasimhan S, Gopalakrishnan V, et al. Pathogen-mediated manipulation of arthropod microbiota to promote infection. Proc Natl Acad Sci U S A. 2017;114(5):E781-E90; doi: 10.1073/pnas.1613422114.

9. Cirimotich CM, Dong Y, Clayton AM, Sandiford SL, Souza-Neto JA, Mulenga M, et al. Natural microbe-mediated refractoriness to Plasmodium Infection in Anopheles gambiae. Science. 2011;332(6031):855-8; doi: 10.1126/science.1201618.

10. Dong Y, Manfredini F, Dimopoulos G. Implication of the mosquito midgut microbiota in the defense against malaria parasites. PLOS Pathog. 2009;5(5); doi: 10.1371/journal.ppat.1000423.

11. Gall CA, Reif KE, Scoles GA, Mason KL, Mousel M, Noh SM, et al. The bacterial microbiome of Dermacentor andersoni ticks influences pathogen susceptibility. ISME Journal. 2016;10(8):1846-55; doi: 10.1038/ismej.2015.266.

12. Narasimhan S, Rajeevan N, Liu L, Zhao YO, Heisig J, Pan JY, et al. Gut microbiota of the tick vector Ixodes scapularis modulate colonization of the Lyme disease spirochete. Cell Host \& Microbe. 2014;15(1):58-71; doi: 10.1016/j.chom.2013.12.001.

13. Li LH, Zhang Y, Zhu D, Zhou XN. Endosymbionts alter larva-to-nymph transstadial transmission of Babesia microti in Rhipicephalus haemaphysaloides ticks. Frontiers in Microbiology. 2018;9; doi: 10.3389/fmicb.2018.01415.

14. McMeniman CJ, Lane RV, Cass BN, Fong AWC, Sidhu M, Wang YF, et al. Stable introduction of a life-shortening Wolbachia infection into the mosquito Aedes aegypti. Science. 2009;323(5910):141-4; doi: 10.1126/science.1165326.

468

15. Bian GW, Xu Y, Lu P, Xie Y, Xi ZY. The endosymbiotic bacterium Wolbachia induces resistance to dengue virus in Aedes aegypti. PLOS Pathog. 2010;6(4); doi:

10.1371/journal.ppat.1000833. 
16. Kambris Z, Cook PE, Phuc HK, Sinkins SP. Immune activation by life-shortening Wolbachia and reduced filarial competence in mosquitoes. Science. 2009;326(5949):1346; doi: 10.1126/science.1177531.

17. Moreira LA, Iturbe-Ormaetxe I, Jeffery JA, Lu G, Pyke AT, Hedges LM, et al. A Wolbachia symbiont in Aedes aegypti limits infection with dengue, chikungunya, and Plasmodium. Cell. 2009;139(7):1268-78; doi: 10.1016/j.cell.2009.11.042.

18. Hoffmann AA, Montgomery BL, Popovici J, Iturbe-Ormaetxe I, Johnson PH, Muzzi F, et al. Successful establishment of Wolbachia in Aedes populations to suppress dengue transmission. Nature. 2011;476(7361):454-U107; doi: 10.1038/nature10356.

19. Walker T, Johnson PH, Moreira LA, Iturbe-Ormaetxe I, Frentiu FD, McMeniman CJ, et al. The wMel Wolbachia strain blocks dengue and invades caged Aedes aegypti populations. Nature. 2011;476(7361):450-U101; doi: 10.1038/nature10355.

20. Dantas-Torres F, Chomel BB, Otranto D. Ticks and tick-borne diseases: a One Health perspective. Trends Parasitol. 2012;28(10):437-46; doi: 10.1016/j.pt.2012.07.003.

21. Kurtenbach K, Hanincova K, Tsao JI, Margos G, Fish D, Ogden NH. Fundamental processes in the evolutionary ecology of Lyme borreliosis. Nat Rev Microbiol. 2006;4:660-9.

22. Lindquist L, Vapalahti O. Tick-borne encephalitis. Lancet. 2008;371(9627):1861-71; doi: 10.1016/s0140-6736(08)60800-4.

23. Stuen S, Granquist EG, Silaghi C. Anaplasma phagocytophilum-a widespread multi-host pathogen with highly adaptive strategies. Front Cell Infect Microbiol. 2013;3; doi: 10.3389/fcimb.2013.00031.

24. Gasmi S, Ogden NH, Lindsay L, Burns S, Fleming S, Badcock J, et al. Surveillance for Lyme disease in Canada: 2009-2015. Canada Communicable Disease Report. 2017;43(10):194-9.

25. Godfrey ER, Randolph SE. Economic downturn results in tick-borne disease upsurge. Parasit Vectors. 2011;4; doi: 10.1186/1756-3305-4-35.

26. Mysterud A, Easterday WR, Stigum VM, Aas AB, Meisingset EL, Viljugrein H. Contrasting emergence of Lyme disease across ecosystems. Nature Communications. 2016;7; doi: 10.1038/ncomms11882.

27. Mysterud A, Jore S, Østerås O, Viljugrein H. Emergence of tick-borne diseases at northern latitudes in Europe: a comparative approach. Scientific Reports. 2017;7(1):16316; doi: 10.1038/s41598-017-15742-6.

28. Ogden NH, Lindsay LR, Morshed M, Sockett PN, Artsob H. The emergence of Lyme disease in Canada. Canadian Medical Association Journal. 2009;180(12):1221-4; doi: 10.1503/cmaj.080148.

29. van Duijvendijk G, Sprong H, Takken W. Multi-trophic interactions driving the transmission cycle of Borrelia afzelii between Ixodes ricinus and rodents: a review. Parasit Vectors. 2015;8:1-11; doi: 10.1186/s13071-015-1257-8.

30. Belli A, Sarr A, Rais O, Rego ROM, Voordouw MJ. Ticks infected via co-feeding transmission can transmit Lyme borreliosis to vertebrate hosts. Scientific Reports. 2017;7(1):5006; doi: 10.1038/s41598-017-05231-1.

31. Jacquet M, Genné D, Belli A, Maluenda E, Sarr A, Voordouw MJ. The abundance of the Lyme disease pathogen Borrelia afzelii declines over time in the tick vector Ixodes ricinus. Parasit Vectors. 2017;10(1):257; doi: 10.1186/s13071-017-2187-4. 
32. Jacquet M, Margos G, Fingerle V, Voordouw MJ. Comparison of the lifetime host-to-tick transmission between two strains of the Lyme disease pathogen Borrelia afzelii. Parasit Vectors. 2016;9; doi: 10.1186/s13071-016-1929-z.

33. Herlemann DPR, Labrenz M, Jurgens K, Bertilsson S, Waniek JJ, Andersson AF. Transitions in bacterial communities along the $2000 \mathrm{~km}$ salinity gradient of the Baltic Sea. Isme Journal. 2011;5(10):1571-9; doi: 10.1038/ismej.2011.41.

34. Oksanen J, Blanchet FG, Friendly M, Kindt R, Legendre P, McGlinn D, et al.: vegan: Community Ecology Package. R package version 2.5-6. https://CRAN.Rproject.org/package=vegan. In.; 2019.

35. Bates D, Machler M, Bolker BM, Walker SC. Fitting Linear Mixed-Effects Models Using lme4. Journal of Statistical Software. 2015;67(1):1-48; doi: 10.18637/jss.v067.i01.

36. Gu ZG, Eils R, Schlesner M. Complex heatmaps reveal patterns and correlations in multidimensional genomic data. Bioinformatics. 2016;32(18):2847-9; doi: 10.1093/bioinformatics/btw313.

37. McMurdie PJ, Holmes S. phyloseq: An R Package for Reproducible Interactive Analysis and Graphics of Microbiome Census Data. PLOS ONE. 2013;8(4); doi: 10.1371/journal.pone.0061217.

38. Li W, Chang Y. CD-HIT-OTU-MiSeq, an Improved Approach for Clustering and Analyzing Paired End MiSeq 16S rRNA Sequences. bioRxiv. 2017:153783; doi: 10.1101/153783.

39. Quast C, Pruesse E, Yilmaz P, Gerken J, Schweer T, Yarza P, et al. The SILVA ribosomal RNA gene database project: improved data processing and web-based tools. Nucleic Acids Res. 2013;41(D1):D590-D6; doi: 10.1093/nar/gks1219.

40. Bolger AM, Lohse M, Usadel B. Trimmomatic: a flexible trimmer for Illumina sequence data. Bioinformatics. 2014;30(15):2114-20; doi: 10.1093/bioinformatics/btu170.

41. Bengtsson-Palme J, Hartmann M, Eriksson KM, Pal C, Thorell K, Larsson DGJ, et al. METAXA2: improved identification and taxonomic classification of small and large subunit rRNA in metagenomic data. Molecular Ecology Resources. 2015;15(6):1403-14; doi: 10.1111/1755-0998.12399.

42. Binetruy F, Dupraz M, Buysse M, Duron O. Surface sterilization methods impact measures of internal microbial diversity in ticks. Parasit Vectors. 2019;12:10; doi: 10.1186/s13071-019-3517-5.

43. Salter SJ, Cox MJ, Turek EM, Calus ST, Cookson WO, Moffatt MF, et al. Reagent and laboratory contamination can critically impact sequence-based microbiome analyses. Bmc Biology. 2014;12; doi: 10.1186/s12915-014-0087-z.

44. Heise SR, Elshahed MS, Little SE. Bacterial diversity in Amblyomma americanum (Acari: Ixodidae) with a focus on members of the genus Rickettsia. J Med Entomol. 2010;47(2):258-68; doi: 10.1603/me09197.

45. Zolnik CP, Prill RJ, Falco RC, Daniels TJ, Kolokotronis S-O. Microbiome changes through ontogeny of a tick pathogen vector. Mol Ecol. 2016;25(19):4963-77; doi: $10.1111 /$ mec. 13832 .

46. Diuk-Wasser MA, Hoen AG, Cislo P, Brinkerhoff R, Hamer SA, Rowland M, et al. Human risk of infection with Borrelia burgdorferi, the Lyme disease agent, in Eastern United States. Am J Trop Med Hyg. 2012;86(2):320-7; doi: 10.4269/ajtmh.2012.110395. 
47. Salem H, Florez L, Gerardo N, Kaltenpoth M. An out-of-body experience: the extracellular dimension for the transmission of mutualistic bacteria in insects. P Roy Soc B-Biol Sci. 2015;282(1804); doi: 10.1098/rspb.2014.2957.

48. Glader O, Puljula E, Jokioja J, Karonen M, Sinkkonen J, Hytonen J. NMR metabolome of Borrelia burgdorferi in vitro and in vivo in mice. Scientific Reports. 2019;9; doi: 10.1038/s41598-019-44540-5.

49. LaRocca TJ, Benach JL. The important and diverse roles of antibodies in the host response to Borrelia infections. Curr Top Microbiol Immunol. 2008;319:63-103.

50. Tracy KE, Baumgarth N. Borrelia burgdorferi manipulates innate and adaptive immunity to establish persistence in rodent reservoir hosts. Frontiers in Immunology. 2017;8; doi:

10.3389/fimmu.2017.00116.

51. Wooten RM, Weis JJ. Host-pathogen interactions promoting inflammatory Lyme arthritis: use of mouse models for dissection of disease processes. Curr Opin Microbiol. 2001;4(3):274-9; doi: 10.1016/s1369-5274(00)00202-2.

52. Kraiczy P. Travelling between two worlds: complement as a gatekeeper for an expanded host range of Lyme disease spirochetes. Veterinary Sciences. 2016;3(2):12.

53. Kang IS, Barthold SW, Persing DH, Bockenstedt LK. T-helper-cell cytokines in the early evolution of murine Lyme arthritis. Infect Immun. 1997;65(8):3107-11.

54. Schwanz LE, Voordouw MJ, Brisson D, Ostfeld RS. Borrelia burgdorferi has minimal impact on the Lyme disease reservoir host Peromyscus leucopus. Vector-Borne Zoonot. 2011;11(2):117-24; doi: 10.1089/vbz.2009.0215.

55. Seller KP, Vavrin Z, Eichwald E, Hibbs JB, Weis JJ. Nitric oxide production during murine Lyme disease - lack of involvement in host-resistance or pathology. Infect Immun. 1995;63(10):3886-95. 


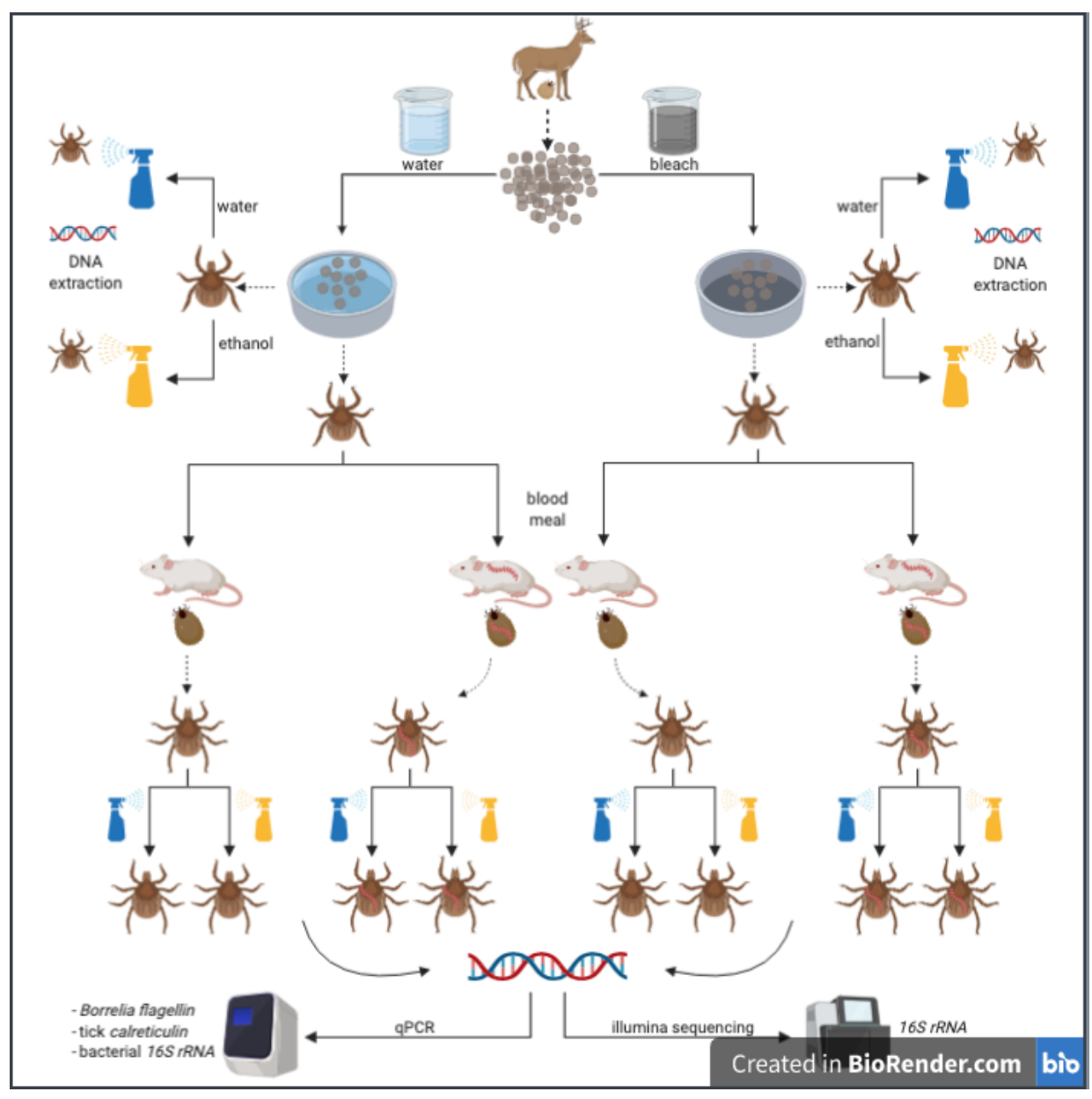

Figure 1. Experimental design. Engorged female I. ricinus ticks $(n=10)$ were collected from roe deer captured in the Chizé forest, France and laid their eggs in the laboratory. Each of the 10 egg batches was split into two batches and rinsed with either $10 \%$ bleach $(n=10$ batches) or distilled water ( $\mathrm{n}=10$ batches) and hatched into larvae. To determine whether the egg bleaching treatment reduced the microbiome, a subset of larvae was tested using qPCR and Illumina sequencing of the bacterial $16 S$ rRNA gene. Larvae for each of the 20 batches were split into two groups of $\sim 100$ larvae. For each of the 20 batches of eggs (10 tick families x 2 egg washing treatments), one group of larvae was fed on an uninfected control mouse ( $\mathrm{n}=20$ control mice) whereas the other group of larvae was fed on a B. afzelii-infected mouse $(n=20$ infected mice). Engorged larvae were placed in individual Eppendorf tubes to moult into nymphs. Four weeks after the moult, 10 nymphs were randomly selected from each of the 40 mice and frozen at $80^{\circ} \mathrm{C}(\mathrm{n}=400$ nymphs). These nymphs were tested for $B$. afzelii infection using qPCR and for their bacterial load and microbiome using qPCR and Illumina sequencing of the bacterial $16 \mathrm{~S}$ $r R N A$ gene. 

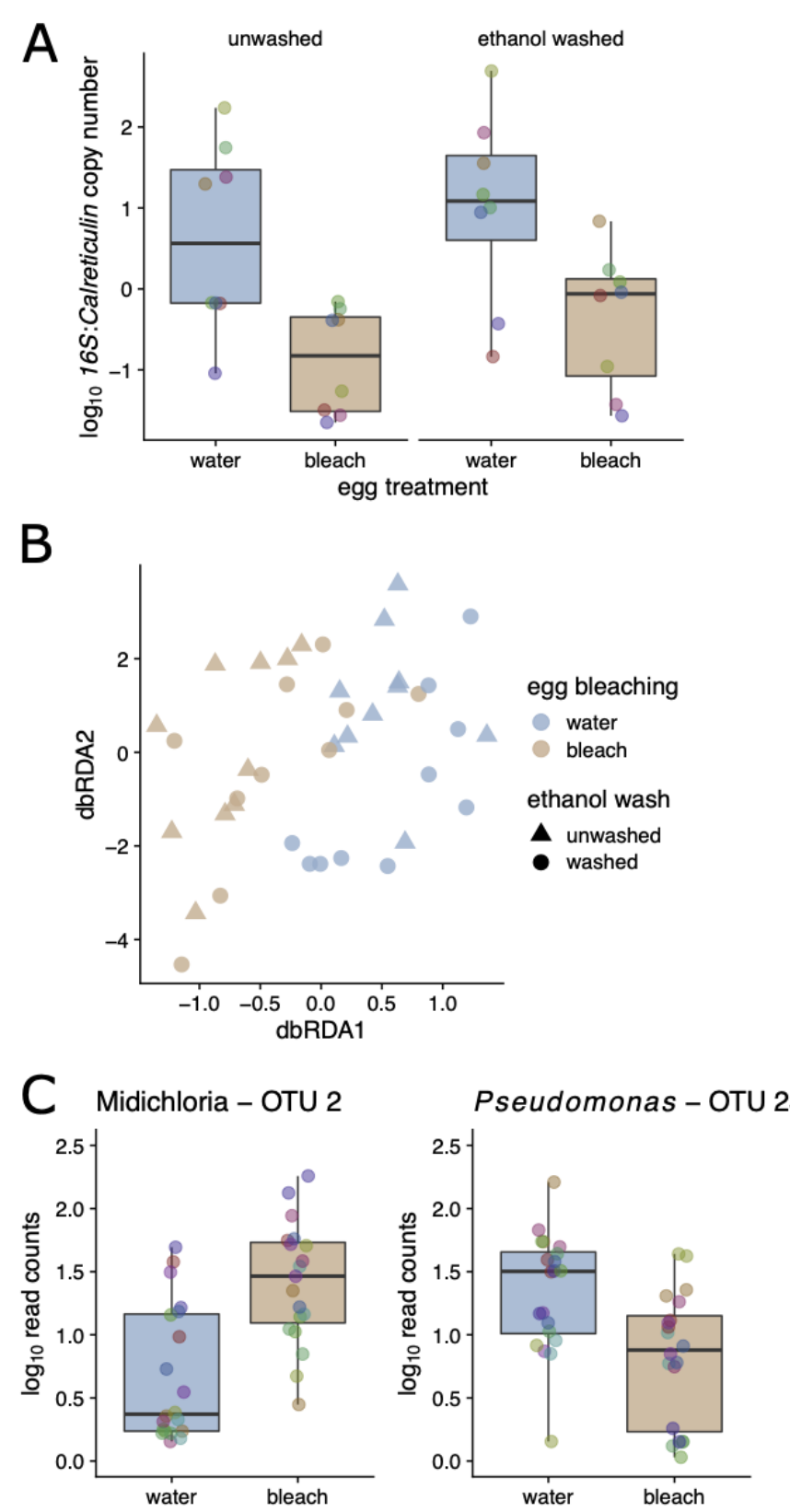

608

egg treatment

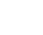


618 reads). Colored points in boxplots represent individual data points (pooled larval samples, 619 colored by the tick mother; 'family'; $\mathrm{N}=10$ ).
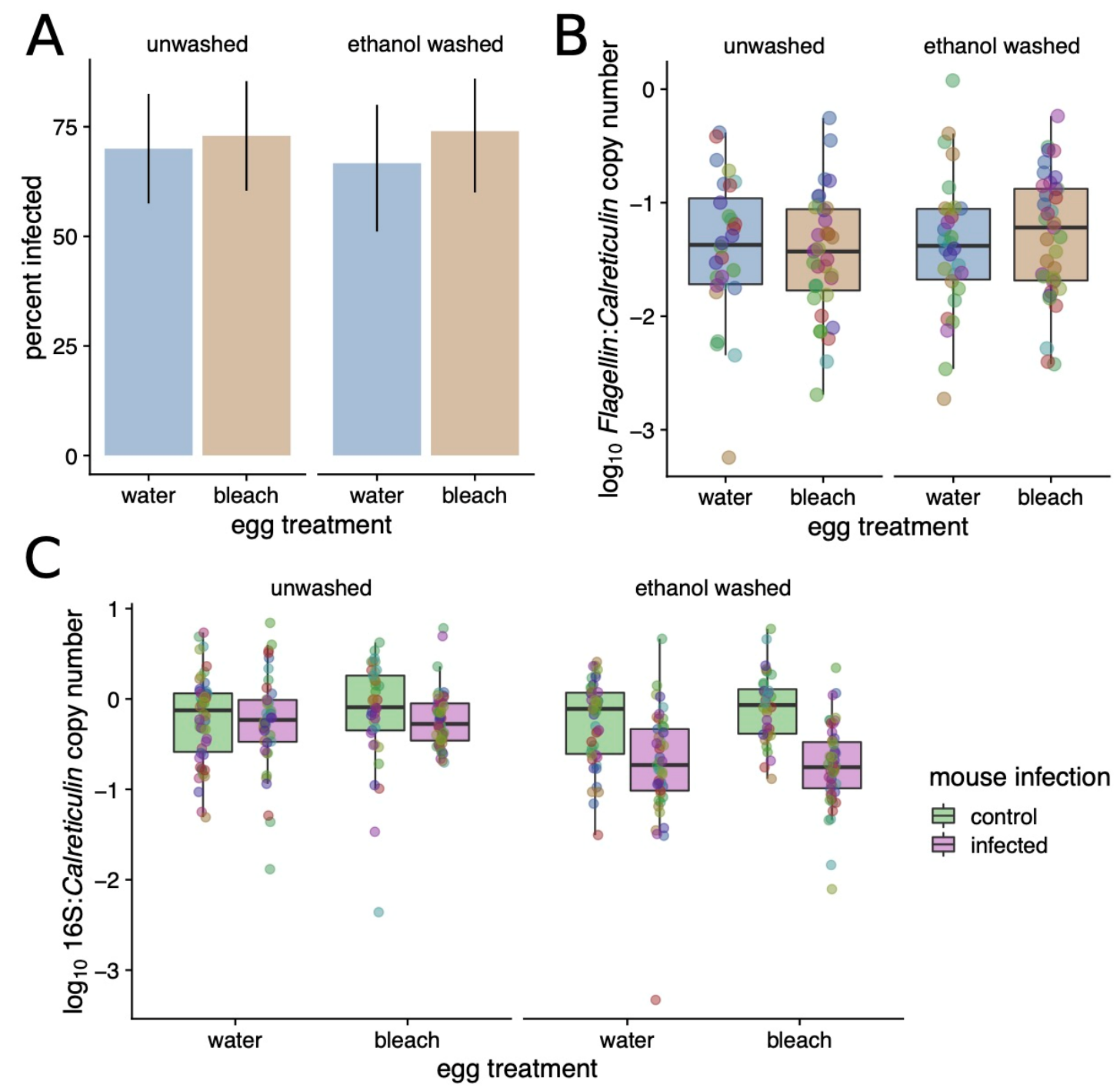

mouse infection

追 control

官 infected

624 Figure 3. 'Dysbiosing' larval ticks does not affect infection success by B. afzelii. Neither A. the percentage of nymphs that acquired $B$. afzelii during their larval blood meal nor B. the inferred $B$. afzelii load in these nymphs were affected by prior microbiome disruption (all $\mathrm{P}>>$ 0.05). C. Feeding on B. afzelii-infected mice decreased the bacterial load in I. ricinus nymphs; this effect was more visible in the nymphs that were washed prior to DNA extraction compared

629 to the unwashed nymphs. 

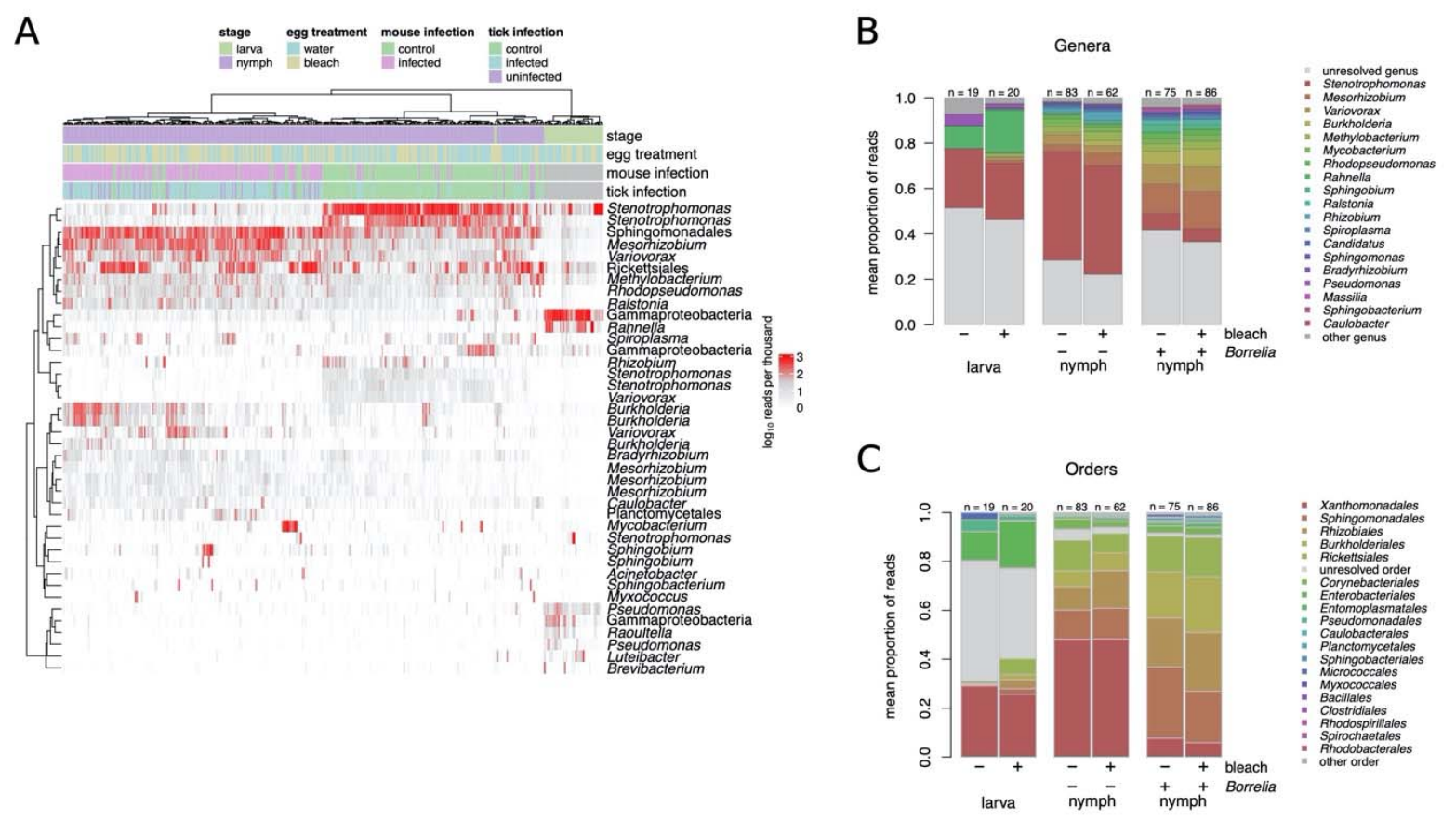

631 Figure 4. Life history stage and B. afzelii exposure affect tick microbiome. A. Heatmap of number of reads assigned $\left(\log _{10}(x+1)\right.$ per thousand) for top 40 OTUs across all samples in the experiment. Highest taxonomy reliably assigned by Metaxa2 is shown. Dendrograms are based on hierarchical clustering of Bray-Curtis dissimilarities using Ward's method. B. Composition of 635 treatment groups and life histories, with top 40 OTUs aggregated (as mean of samples per group) at the genus level. C. As above, at the order level. 
A

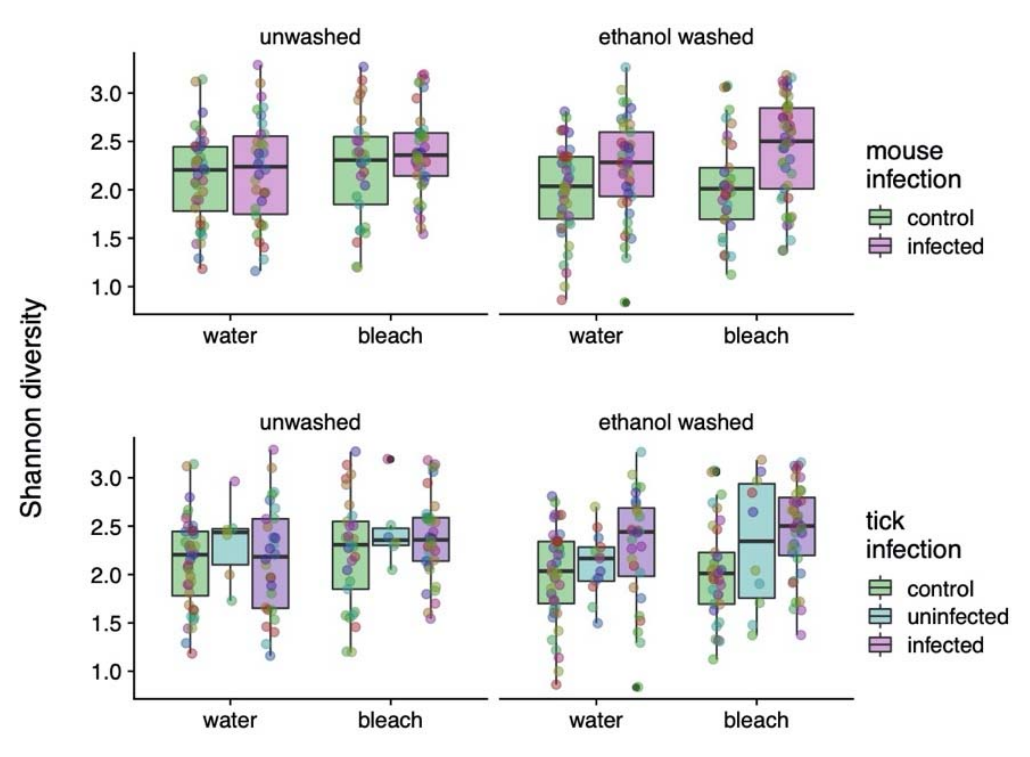

egg treatment

C
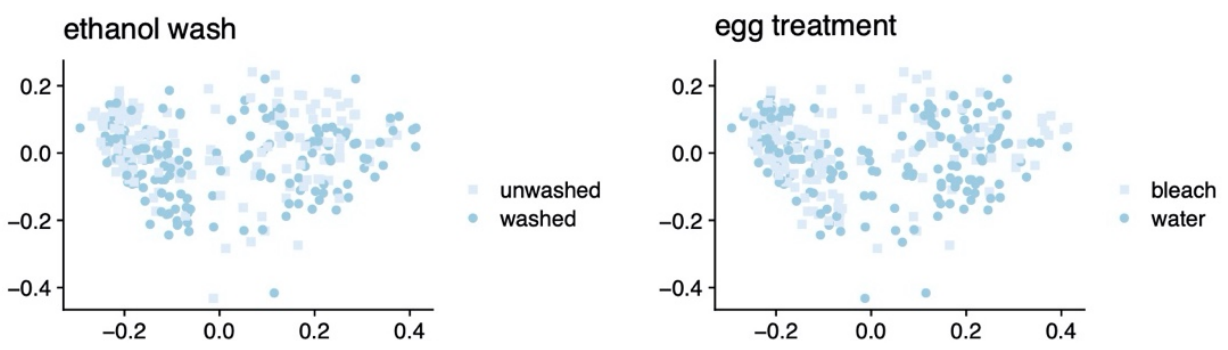

잉
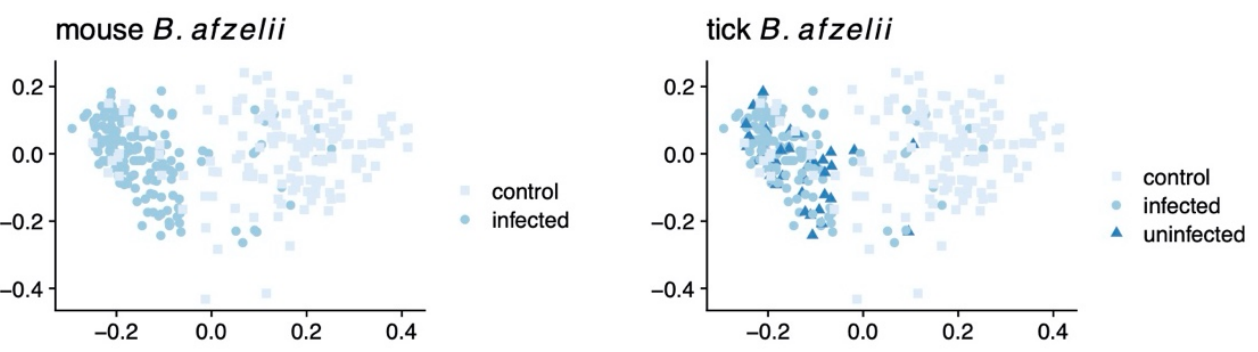

PCoA1

638 Figure 5. Egg bleaching and rinsing and B. afzelii exposure increase the diversity of the $16 \mathrm{~S}$ 639 microbiome of I ricinus nymphs. Shannon diversity increases in both A. B. afzelii exposed 640 ticks and B. B. afzellii infected ticks (P <0.01). Akaike's Information Criterion (AIC) shows 641 exposure to be a stronger predictor of Shannon diversity than infection (deltaAIC $\sim 6$ ) in linear 642 mixed models. Points represent individual tick nymphs colored by family of origin. C. Principal 643 Coordinates Analysis of nymphal 16S counts, colored by experimental factors; $B$ afzelii exposure 644 best stratifies groups on PCoA 1 (deltaAIC $\sim 11$ vs infection). 


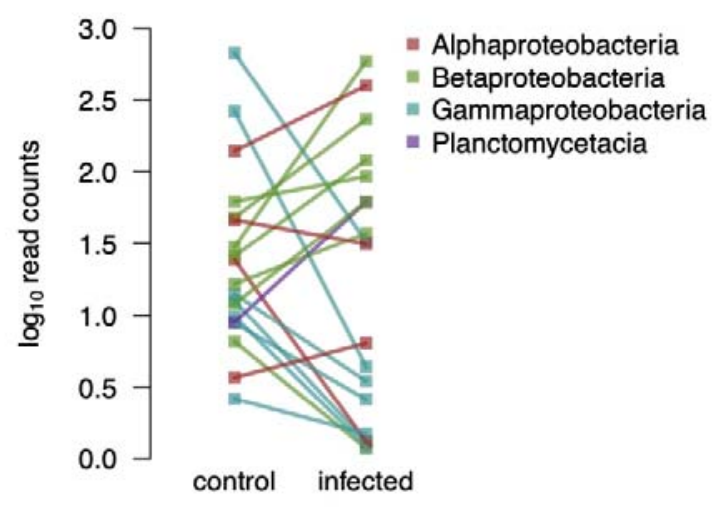

648 Figure 6. Proportional abundance of taxa that changed significantly in I. ricinus nymphs (19/40 649 at $\left.\mathrm{P}_{\mathrm{adj}}<0.05\right)$ in response to feeding on B. afzelii infected mice, shown at the level of class.

650 There is significant taxonomic dependence (Fisher's exact test $\mathrm{P}=0.004$ ) of taxonomic response. 


\section{Supplemental Material:}

654

655 Data S0: Description of molecular methods

656

657

658

659

Data S1: Metadata

660

Data S2: Metadata

661

662

663

Data S3: OTU table produced by CD-HIT-OTU_MiSEQ.

664

665

Data S4: OTU clusters produced by CD-HIT-OTU_MiSEQ.

666

667 